\title{
Postoperative chest tube placement after thoracoscopic wedge resection of lung for primary spontaneous pneumothorax: is it mandatory?
}

\author{
Jae Bum Park, Jae Joon Hwang, Woo Surng Lee, Yo Han Kim, Song Am Lee \\ Department of Thoracic and Cardiovascular Surgery, Konkuk University Medical Center, Seoul, Korea \\ Contributions: (I) Conception and design: SA Lee, JB Park; (II) Administrative support: SA Lee; (III) Provision of study materials or patients: JB \\ Park; (IV) Collection and assembly of data: JB Park, WS Lee; (V) Data analysis and interpretation: JJ Hwang, YH Kim; (VI) Manuscript writing: All \\ authors; (VII) Final approval of manuscript: All authors. \\ Correspondence to: Song Am Lee, MD. Department of Thoracic and Cardiovascular Surgery, Konkuk University Medical Center, 120-1 Neungdong- \\ ro, Gwangiin-gu, Seoul, Korea. Email: azzy@kuh.ac.kr.
}

\begin{abstract}
Background: The goal of present study is to assess the efficiency and feasibility of postoperative care without positioning of chest tube after video-assisted thoracoscopic surgery (VATS) in patients with primary spontaneous pneumothorax (PSP).

Methods: We conducted a prospective randomized trial of patients who have undergone video-assisted thoracoscopic wedge resection surgery for PSP. During the period of April 2015 until December 2016, 119 eligible patients undergoing VATS for PSP were consecutively included. We divided the patients into two groups at random: (I) avoiding chest tube (ACT) group, comprising 58 patients in whom chest tube was not placed intra or postoperatively, and (II) indwelling chest tube (ICT) group, comprising 61 patients in whom chest tube was placed conventionally. Primary end point was to evaluate and analyze the postoperative clinical data and relevant morbidities between two groups.

Results: The mean postoperative length of hospital stay ( $3.57 \pm 0.79$ vs. $5.39 \pm 0.97$ days) and postoperative pain $(1.37 \pm 0.78$ vs. $2.53 \pm 0.63)$ in ACT group were significantly lower than in the ICT group $(\mathrm{P}<0.05)$. However, there were no statistical differences between two groups regarding other clinical data and postoperative morbidities. There were no patients who experienced significant adverse events postoperatively. Conclusions: Postoperative management without chest tube placement is safe and feasible approach for patients undergoing thoracoscopic wedge resection for PSP.
\end{abstract}

Keywords: Primary spontaneous pneumothorax (PSP); chest tube management; thoracoscopic surgery; wedge resection of lung; postoperative management

Submitted Mar 18, 2018. Accepted for publication Jul 01, 2018.

doi: $10.21037 /$ jtd.2018.07.13

View this article at: http://dx.doi.org/10.21037/jtd.2018.07.13

\section{Introduction}

Conventionally, we routinely have placed a chest tube in the pleural cavity after lung resection surgery even though air leak is not identified in the middle of operation. It will only be removed when no air leak is detected and the daily pleural effusion has decreased below a certain volume. Inserted chest drain may commonly cause pain, infectious problems, and prolonged length of stay (LOS).
The duration of chest tube insertion is known to be one of main factors influencing hospitalization period and relevant costs. Therefore, early removal of chest tube is believed to increase patient's satisfaction and quality of life, and potentially decrease the risk of pulmonary complications (1). Another similar research was also carried out to assess whether early removal of chest tube was justified within 2 hours after video-assisted thoracoscopic 
surgery (VATS) (2). Recently, surgical instrument and devices used in VATS have improved considerably, resulting in less injury to the chest wall than usual thoracotomy wound. Besides, the unexpected adverse events such as parenchymal air leaks from the stapler line appears to be uncommon on the strength of advances in surgical equipment and technique, but excluding in patients with severe emphysematous and diffuse bullous changes of lung, or inappropriate usage of stapler. Several previous researches have described that postoperative management without chest tube after thoracoscopic wedge resection surgery is safe and beneficial for patients, and it may contribute to an early recovery $(3,4)$. However, these studies have some limitations characterized by retrospective review with only patients group within strict established criteria. Compared to other lung surgery using the same thoracoscopic technique, pneumothorax is relatively simple, and it takes shorter operation time. In this respect, we have come to doubt on the justification for routine postoperative chest tube placement. In present study, a prospective randomized controlled trial was performed to analyze whether postoperative treatment without chest tube placement is justified in thoracoscopic wedge resection surgery for primary spontaneous pneumothorax (PSP). The purpose of this study is to determine the validity and feasibility of avoiding chest drains following VATS wedge resection for PSP.

\section{Methods}

\section{Study design and patients}

During the period of April 2015 until December 2016, 119 eligible patients undergoing VATS for PSP were included. We made a plan to perform a prospective randomized trial of patients who underwent VATS wedge resection of lung for PSP. The patients were randomly allocated to the two groups: (I) avoiding chest tube (ACT) group, comprising 58 patients in whom chest tube was not placed intra or postoperatively, and (II) indwelling chest tube (ICT) group, comprising 61 patients in whom chest tube was placed conventionally. Randomization was done as the eligible patients entered the study with odd number assigned to ACT placement management, and even number for traditional chest tube placement management postoperatively. All patients undergoing VATS wedge resection of lung for PSP were considered for inclusion in the study. Patients who refused to participate in the study, and aged $<15$ or $>70$ years old were excluded from the study. Besides, patients with a previous ipsilateral thoracic surgery and secondary spontaneous pneumothorax (SSP) associated with underlying lung diseases such as pulmonary tuberculosis, empyema, cystic fibrosis, and chronic obstructive pulmonary disease (COPD) were also excluded (CONSORT diagram) (Figure 1). Informed consent was naturally obtained from all patients included in present study. The study was approved by Institutional Review Board of Konkuk University Medical Center (KUH1080026). This trial was reported in accordance with the Consolidated Standards of Reporting Trials (CONSORT) statement. The clinical data including patient's basic characteristics, and postoperative morbidity and other results were evaluated and compared between the two groups. Our primary outcome assessed was relative occurrence of postoperative complications such as pneumothorax or pleural effusion which may require chest tube reinsertion. Secondary outcomes included the length of hospital stay, the duration of chest tube placement, requirement of analgesic and other postoperative complications.

\section{Surgical management}

All patients were intubated with a double lumen endobronchial tube or single lumen tube with bronchial blockade to achieve single lung ventilation under general anesthesia. We usually tried to access the intrathoracic area through the 7 th intercostal space at the anterior midaxillary line for exploration of the lung. A $5-\mathrm{mm}, 30^{\circ}$ operating thoracoscope (Karl Storz, Tuttlingen, Germany) was routinely used to explore the pleural space. First, exploratory thoracoscopy was undertaken, and the pleural space was inspected with this thoracoscope. Subsequently, another two access ports were established for grasping the lung and switching the port for the thoracoscope. Finally, an endoscopic liner stapler (Echelon Endostapler, Ethicon, Cincinnati, OH, USA) was inserted to perform wedge resection for removing bulla and/or blebs. After termination of wedge resection of lung, we also performed pleural abrasion with sterile gauze soaked in betadine solution (containing a mixture of $10 \%$ iodopovidone, $20 \mathrm{~mL}$ and normal saline, $80 \mathrm{~mL}$ ), which can induce the effect of chemical and mechanical pleurodesis at the same time. Upon completion of the procedure, a 28 French silicon chest tube (Covidien, Minneapolis, MN, USA) was routinely placed in the pleural space in the ICT group, while chest drain removal was done after full deairing 


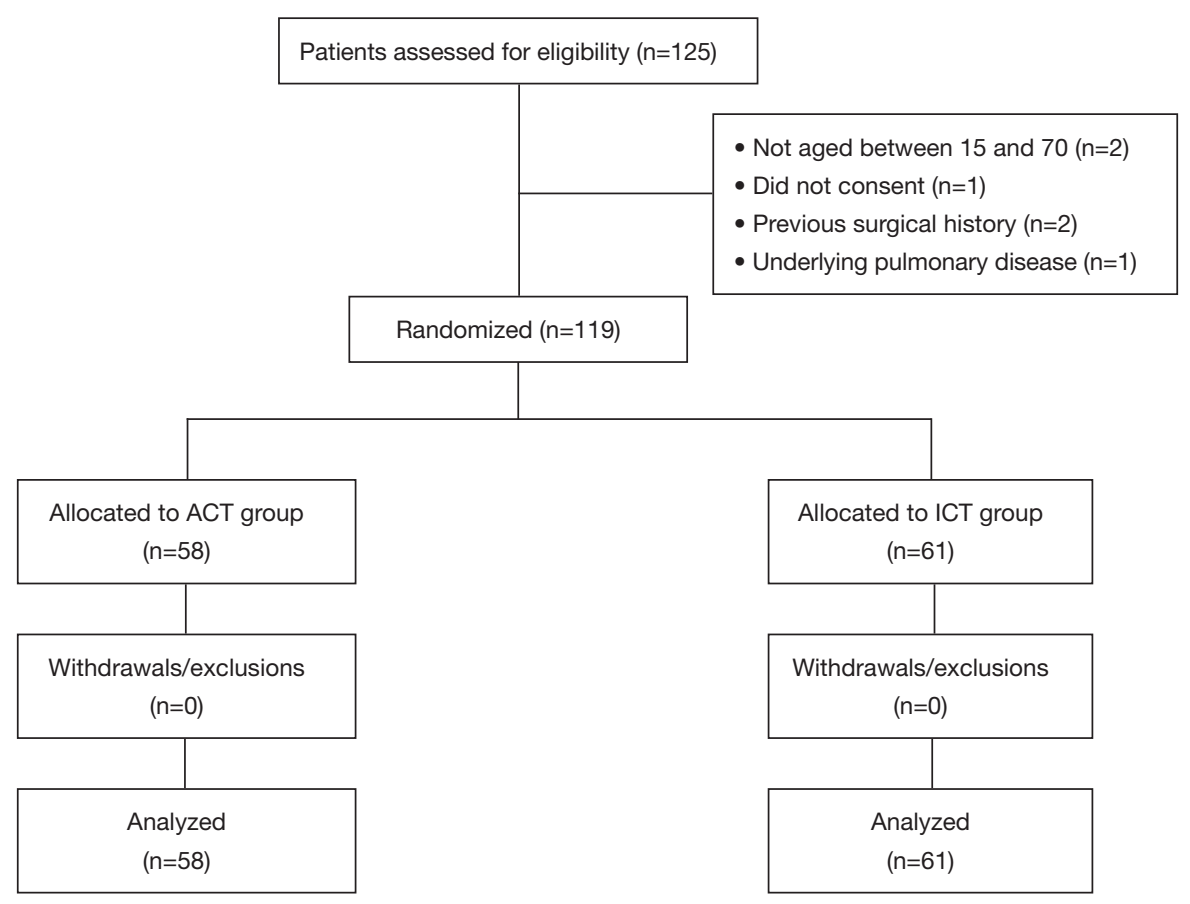

Figure 1 Consort diagram of the study. ACT, avoiding chest tube; ICT, indwelling chest tube.

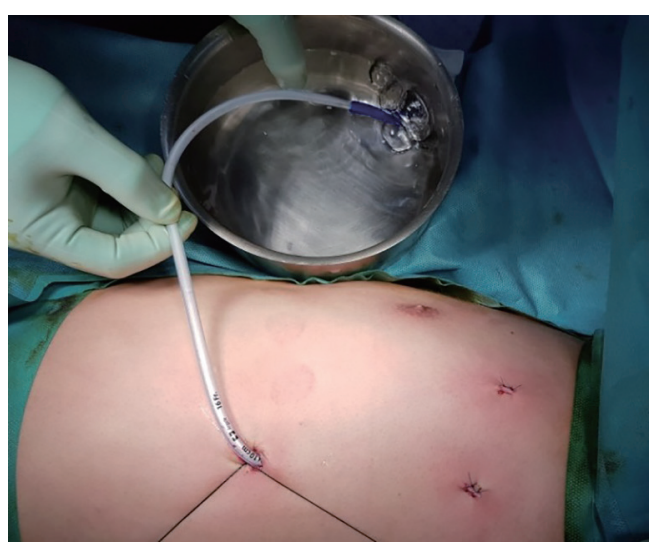

Figure 2 In ACT group, air evacuation process is achieved by temporarily placed small bore chest tube $(16 \mathrm{Fr})$ into the pleural cavity with a bowl of aseptic water (the water seal system). Chest tube is removed after full deairing and confirmation of no air leak. ACT, avoiding chest tube.

through the water sealing test in the ACT group.

\section{Deairing and water sealing test}

An alternative way of water sealing test to detect air leaks has been previously described (3). According to our manner of deairing procedure, a 16 French chest drain tube (Covidien, Minneapolis, MN, USA) was inserted apically through the thoracoscopic port, and ventilation was converted into two lung ventilations. Then, we closely observed the re-expansion of the lung with thoracoscope through the other port. The chest tube was temporarily located and fixed by tagging sutures, and the tip was stuck in the sterile water with a bowl during two lung ventilations. The drain tip was inspected to ensure air evacuation and full deairing of lung (Figure 2). After confirming that there is no air leakage while the lung was fully inflated (maximum airway pressure of $25 \mathrm{mmHg}$ ), the chest drain was removed with keeping a positive inspiratory pressure, and we immediately closed the remnant wounds with subcutaneous sutures.

\section{Postoperative management}

A simple chest radiography examination was conducted shortly after the transfer to the recovery room in all 119 patients after surgery. Patients were restricted in their any oral intake for 4 hours postoperatively, after that routine medication for pain control was given three times a day as acetaminophen $325 \mathrm{mg}$ with tramadol $37.5 \mathrm{mg}$. An intramuscular injection (diclofenac $90 \mathrm{mg}$ ) with a minimum 
Table 1 Clinical data

\begin{tabular}{lccc}
\hline Characteristics & ACT group $(n=58)$ & ICT group $(n=61)$ & $P$ \\
\hline Age (years) & $22.76 \pm 6.06$ & $29.23 \pm 14.03$ & 0.002 \\
Sex (male/female) & $53 / 5$ & $55 / 6$ & 0.821 \\
Side (right/left) & $29 / 29$ & $30 / 31$ & 0.930 \\
Operation time (min) & $55.81 \pm 7.20$ & $56.57 \pm 8.43$ & 0.596 \\
Number of specimens & $1.10 \pm 0.30$ & $1.11 \pm 0.32$ & 0.845 \\
Number of cartridges & $2.33 \pm 0.63$ & $2.36 \pm 0.63$ & 0.776 \\
\hline
\end{tabular}

Values are presented as mean \pm standard deviation. ACT, avoiding chest tube; ICT, indwelling chest tube.

6-hour interval was provided for patients with unbearable pain. Chest pain or discomfort after operation was assessed and recorded everyday using the visual analogue scale (VAS) from 0 (no pain) to 10 (excruciating pain). All patients routinely had chest radiography exam daily morning. The chest drain tube in the ICT group was connected to a water seal and set to $-20 \mathrm{cmH}_{2} \mathrm{O}$ wall suction care until removal. The chest tube removal criteria were air leak cessation, fully expanded lung on chest radiograph, and the drainage of pleural fluid $<150 \mathrm{~mL} /$ day. We routinely checked the status of air leak every 8 hours. The patients were naturally discharged from the hospital if they had no specific complications. And, they were supposed to have a first visit in the outpatient clinic for radiological examination and other postoperative management in 2 weeks after surgery. Indications for reinsertion of chest drainage tube were expanding aggravated pneumothorax, considerable amount of pleural effusion, or expanding massive subcutaneous emphysema.

\section{Statistical analysis}

Categorical variables were expressed as percentages or numbers, and continuous variables were expressed as means with standard deviations. The Chi-square test or Fisher's exact test was used to compare categorical variables. The Student's $t$-test was used to analyze continuous variables after testing for normality of distribution. The recurrence of pneumothorax within the two groups was calculated using Kaplan-Meier analysis, and log rank test was performed to compare significant difference between the two groups. All $\mathrm{P}$ values less than 0.05 were considered statistically significant. Statistical analyses were performed with SPSS 22.0 (SPSS Inc., Chicago, IL, USA).

\section{Results}

Clinical data are summarized in Table 1. There were no statistically significant differences between the two groups regarding individual characteristics and intraoperative data including operating time, number of specimens and cartridges usage, but excluding age. The overall postoperative outcomes between the two groups are shown in Table 2. Two patients in ACT group required closed thoracostomy after the operation. One patient developed expanding aggravated pneumothorax on the third day after the surgery, while another patient required thoracostomy due to the development of late increased pleural effusion on the second postoperative day. The reason why aggravated pneumothorax occurred postoperatively is believed to be due to the dysfunction of deairing procedure and water sealing air leakage test. We think that the atelectatic lung segment around the area of the stapling line is the main cause of this event. Also, full expansion of lung could not be ensured by thoracoscope in the operation room during the water sealing test with deairing maneuver. In the other case of requiring chest tube, a small amount of ipsilateral pleural effusion was noted in the follow up chest radiograph exam, which is suspected to be hemothorax. It is thought to occur due to excessive intraoperative mechanical pleurodesis and his bleeding tendency by persistent anticoagulation use. These two cases seem to have resulted from initial technical failures. The mean duration of chest tube placement in the ICT group was $4.23 \pm 0.92$ days. In addition, the mean postoperative length of hospital stay $(3.57 \pm 0.79 v s .5 .39 \pm 0.97$ days) and postoperative pain $(1.37 \pm 0.78$ vs. $2.53 \pm 0.63)$ in the ACT group were significantly lower than in the ICT group $(\mathrm{P}<0.05)$. However, there were no statistical differences between two groups with regard to other clinical data, postoperative morbidities and recurrence rates 
Table 2 Postoperative outcomes

\begin{tabular}{|c|c|c|c|}
\hline Outcomes & ACT group $(n=58)$ & ICT group $(n=61)$ & $\mathrm{P}$ \\
\hline PO hospital stay (days) & $3.57 \pm 0.79$ & $5.39 \pm 0.97$ & 0.001 \\
\hline VAS (POD \#1) & $1.37 \pm 0.78$ & $2.53 \pm 0.63$ & 0.001 \\
\hline VAS (POD \#2) & $0.25 \pm 0.44$ & $1.25 \pm 0.41$ & 0.001 \\
\hline Follow up period (days) & $298.51 \pm 194.37$ & $288.32 \pm 154.87$ & 0.753 \\
\hline Recurrence, n (\%) & $2(3.4)$ & $3(4.9)$ & 0.672 \\
\hline $\begin{array}{l}\text { Partial pneumothorax not requiring a chest tube } \\
\text { insertion, } \mathrm{n}(\%)\end{array}$ & $3(5.2)$ & $0(0.0)$ & 0.083 \\
\hline
\end{tabular}

Values are presented as mean \pm standard deviation or number (\%). ACT, avoiding chest tube; ICT, indwelling chest tube; CTP, chest tube placement; PO, postoperative; VAS, visual analogue scale.

during the follow up period. Minimal partial pneumothorax occurred in 3 patients $(5.2 \%)$ in the ACT group just after surgery, but resolved spontaneously within 24 hours. No patients experienced significant adverse events or involved mortalities, postoperatively.

\section{Discussion}

Many surgeons have a question whether postoperative chest tube placement is indispensable process after VATS wedge resection. Actually, the potential benefits of thoracoscopic surgery might be undervalued by unnecessary ICT after surgery. A number of papers have been reported referring to some alternative ways of postoperative care without chest tube and early removal of chest tube management (1-7). Recent remarkable technological development of the devices in thoracoscopic surgery facilitated less bleeding in the stapler line and fewer air leaks when they were manipulated appropriately (8). However, keeping the chest tube in the pleural cavity for a couple of days after surgery is still believed to diminish the incidence of early postoperative adverse events like pneumothorax or unexpected hemothorax. Cerfolio et al. have suggested that the amount of pleural drainage up to $450 \mathrm{~mL} /$ day cannot be prohibition criteria of chest tube removal (9). Several other documents have introduced that the postoperative management of chest tube without suction system might facilitate to decrease the amount of pleural effusion and minor air leaks after surgery $(10,11)$. Postoperative chest tube placement is not essential procedure in order to prevent abrupt pleural effusion or hemothorax. Comprehensive guidelines were prepared for the management of chest tube through cooperation with the Society of Thoracic Surgeon, the American Association for Thoracic Surgery, European Society of Thoracic Surgeons, and the General Thoracic Surgery Club in 2011 (12). But, they have not discussed postoperative care without chest tube placement, unfortunately. In 1998, the study of early chest tube removal within 90 minutes after VATS in 31 patients was reported by Russo et al., which highlighted shorter hospital LOS and similar morbidities rates in the early chest tube removal group comparing to the group of conventional chest tube management (1). In 2006, Fibla $e t a l$. introduced early removal of chest tube within 60 minutes after VATS biopsy in 135 patients (6). From 2000 to 2009, Nakashima et al. reviewed 333 patients undergoing VATS, among whom 132 patients were employed with chest tube removal management intraoperatively, which revealed a shorter hospital LOS (13). However, these previous reports are principally retrospective study, and limited to highly selective patients whom they set strict criteria of excluding patients at potential risks for significant air leak or pleural effusion postoperatively (14). These favorable results data on the basis of previous documented researches for ACT placement after VATS led us to 
initiate a prospective randomized clinical trial to validate our findings. In present study, our results demonstrated that patients undergoing VATS wedge resection for PSP can be treated without routine postoperative chest tube. PSP is defined as that which appears in patients without definite history of previous underlying pathology that may explain the event. Meanwhile, SSP is mainly related to the presence of underlying lung disease, COPD accompanied by emphysematous bullous changes of lung (15). There are actually many surgical treatment methods for PSP, but less invasive thoracoscopic surgical approach is most commonly applied with low morbidity (16). Because it is considered that the rupture of blebs is one of the main underlying pathologic sources of PSP, surgical wedge resection of lung lesion is primarily performed. Blebectomy, or wedge resection of lung may be accomplished alone or combined with additional procedures such as mechanical or chemical pleurodesis, and/or covering over the staple line $(17,18)$. Traditionally, surgeons routinely have placed a chest tube in the pleural cavity after lung resection surgery even though air leak is not identified in the middle of operation. Considering that VATS for PSP is rather simple and not a too much time-consuming surgery compared to other lung diseases, we clinically tried to assess and evaluate the validity of traditional routine chest tube placement postoperatively. In present study, there were only two patients requiring chest tube insertion after surgery in ACT group. One patient had aggravated pneumothorax which is believed to be dysfunction of deairing in water sealing air leak test. It is assumed that partial collapsed segment of the lung around the area of the stapling line is the main reason for this occurrence. The other case of requiring chest tube is due to pleural effusion which appeared to be hemothorax. We presume that excessive intraoperative mechanical pleurodesis has caused it after all. These two events seem to be derived from early technical failures. Overall, the results of present study may validate that postoperative management without chest tube placement does not hinder the safety of VATS for PSP. The presence or absence of postoperative chest tube did not impact significant postoperative adverse events and relevant morbidities in this study. In addition, the mean postoperative length of hospitalization and postoperative pain in ACT group were significantly lower than in the ICT group $(\mathrm{P}<0.05)$. A shorter hospital stay naturally promotes patients resuming their daily lives and saves on medical costs. To our knowledge, this is the first prospective randomized trial of evaluating justifications of postoperative management without chest tube placement in VATS for PSP. The goal of this study is to determine the feasibility and validity of ACT after VATS wedge resection for PSP. However, a large scale multicenter comparative study should be performed to confirm and ensure our findings. We believe that a postoperative management strategy without chest drain tube can be feasible after further variable and intensified studies.

\section{Conclusions}

In conclusion, postoperative management without chest tube placement is safe and feasible approach for patients undergoing thoracoscopic wedge resection for PSP. No major postoperative complications were observed in ACT group patients. In addition, avoiding postoperative chest tube placement may shorten the duration of the postoperative hospitalization, and reduce amount analgesics used.

\section{Acknowledgements}

None

\section{Footnote}

Conflicts of Interest: The authors have no conflicts of interest to declare.

Ethical Statement: The study was approved by Institutional Review Board of Konkuk University Medical Center (KUH1080026) and written informed consent was obtained from all patients.

\section{References}

1. Russo L, Wiechmann RJ, Magovern JA, et al. Early chest tube removal after videoassisted thoracoscopic wedge resection of the lung. Ann Thorac Surg 1998;66:1751-4.

2. Sienel W, Mueller J, Eggeling S, et al. Early chest tube removal after video-assisted thoracoscopic surgery. Results of a prospective randomized study. Chirurg 2005;76:1155-60.

3. Watanabe A, Watanabe T, Ohsawa H, et al. Avoiding chest tube placement after videoassisted thoracoscopic wedge resection of the lung. Eur J Cardiothorac Surg 2004;25:872-6.

4. Ueda K, Hayashi M, Tanaka T, et al. Omitting chest tube 
drainage after thoracoscopic major lung resection. Eur J

Cardiothorac Surg 2013;44:225-9; discussion 229.

5. Satherley LK, Luckraz H, Rammohan KS, et al. Routine placement of an intercostal chest drain during videoassisted thoracoscopic surgical lung biopsy unnecessarily prolongs in-hospital length of stay in selected patients. Eur J Cardiothorac Surg 2009;36:737-40.

6. Fibla JJ, Molins L, Simon C, et al. Early removal of chest drainage after videothoracoscopic lung biopsy. Interact Cardiovasc Thorac Surg 2006;5:581-3.

7. Luckraz H, Rammohan KS, Phillips M, et al. Is an intercostal chest drain necessary after video-assisted thoracoscopic (VATS) lung biopsy? Ann Thorac Surg 2007;84:237-9.

8. Varela G, Jiménez MF, Novoa NM, et al. Postoperative chest tube management: measuring air leak using an electronic device decreases variability in the clinical practice. Eur J Cardiothorac Surg 2009;35:28-31.

9. Cerfolio RJ, Bryant AS. Results of a prospective algorithm to remove chest tubes after pulmonary resection with high output. J Thorac Cardiovasc Surg 2008;135:269-73.

10. Marshall MB, Deeb ME, Bleier JI, et al. Suction vs water seal after pulmonary resection: a randomized prospective study. Chest 2002;121:831-5.

11. Antanavicius G, Lamb J, Papasavas P, et al. Initial chest tube management after pulmonary resection. Am Surg 2005;71:416-9.

Cite this article as: Park JB, Hwang JJ, Lee WS, Kim YH, Lee SA. Postoperative chest tube placement after thoracoscopic wedge resection of lung for primary spontaneous pneumothorax: is it mandatory? J Thorac Dis 2018;10(8):48124818. doi: $10.21037 /$ jtd.2018.07.13
12. Brunelli A, Beretta E, Cassivi SD, et al. Consensus definitions to promote an evidencebased approach to management of the pleural space. A collaborative proposal by ESTS, AATS, STS, and GTSC. Eur J Cardiothorac Surg 2011;40:291-7.

13. Nakashima S, Watanabe A, Mishina T, et al. Feasibility and safety of postoperative management without chest tube placement after thoracoscopic wedge resection of the lung. Surg Today 2011;41:774-9.

14. Koç T, Routledge T, Chambers A, et al. Do patients undergoing lung biopsy need a postoperative chest drain at all? Interact Cardiovasc Thorac Surg 2010;10:1022-5.

15. Sahn SA, Heffner JE. Spontaneous pneumothorax. N Engl J Med 2000;342:868-74.

16. Herrmann D, Klapdor B, Ewig S, et al. Initial management of primary spontaneous pneumothorax with videoassisted thoracoscopic surgery: a 10-year experience. Eur J Cardiothorac Surg 2016;49:854-9.

17. Lee S, Kim HR, Cho S, et al. Staple line coverage after bullectomy for primary spontaneous pneumothorax: a randomized trial. Ann Thorac Surg 2014;98:2005-11.

18. Hirai K, Kawashima T, Takeuchi S, et al. Covering the staple line with a polyglycolic acid sheet after bullectomy for primary spontaneous pneumothorax prevents postoperative recurrent pneumothorax. J Thorac Dis 2015;7:1978-85. 